\title{
Reference values for and interpretation of the Singapore Caregiver Quality of Life Scale: a quantile regression approach
}

Yin Bun Cheung ${ }^{1,2,3^{*}}$ (D), Shirlyn H. S. Neo ${ }^{4}$, Grace M. Yang ${ }^{4,5}$, Irene Teo ${ }^{4,5}$, Geok Ling Lee ${ }^{6}$, Debra L. M. Qu ${ }^{4}$, Audrey R. X. Koh ${ }^{4}$, Julian Thumboo ${ }^{1,7}$ and Hwee Lin Wee

\begin{abstract}
Purpose: The Singapore Caregiver Quality of Life Scale (SCQOLS) was recently developed and validated in two languages - English and Chinese. The total and domain scores are scaled to range from 0 to 100. However, the scale is not at the interval-ratio level of measurement. To facilitate interpretation, we established the percentiles of the scale's total and domain scores among family caregivers of patients with advanced cancers and demonstrate the effect size in terms of differences in relation to caregiver and patient characteristics.

Methods: Data were drawn from a cross-sectional survey of family caregivers of patients with stage III or IV solid cancers in Singapore. Quantile regression was used to estimate the percentiles in relation to caregiver and patient characteristics.

Results: Participants in adjacent categories of patient's performance status and caregiver's having other family members to share caregiving duties differed by 3 to 5 points in median quality of life total score and most domain scores (each Bonferroni-adjusted P, P[B], <0.05). Ethnicity was associated with the Physical Well-being and Experience \& Meaning domain scores (each $\mathrm{P}[\mathrm{B}]<0.05$ ), with variable direction and magnitude. Education was associated with Mental Well-being and Financial Well-being (each $P[B]<0.05$ ). Equations and examples for calculation of the percentiles are provided.
\end{abstract}

Conclusion: Percentiles and effect size estimates are provided to facilitate interpretation of the SCQOLS.

Keywords: Caregivers, Effect size, Reference values, Singapore Caregiver Quality of Life Scale

\section{Introduction}

Cancer is a disease that imposes major burden not only on patients but also on their family caregivers. There has been a shortage of caregiver quality of life (QOL) measurement scales [1, 2]. Our qualitative study of family caregivers of advanced cancer patients in Singapore, a multi-ethnic society in South-East Asia, has shown substantial differences between the

\footnotetext{
* Correspondence: yinbun.cheung@duke-nus.edu.sg

'Program in Health Services \& Systems Research, Duke-NUS Medical School,

20 College Road, Singapore 169856, Singapore

${ }^{2}$ Centre for Quantitative Medicine, Duke-NUS Medical School, Level 6,

Academia, 20 College Road, Singapore 169856, Singapore

Full list of author information is available at the end of the article
}

concerns of the caregivers and the contents of existing QOL measurement scales developed in the West [3]. A study of the Chinese translation of the Caregiver Quality Of Life Index-Cancer conducted in China also concluded that there was "only partial support for the relevance and construct validity of the scale for Chinese caregivers" [4].

Recently we developed and validated the Singapore Caregiver Quality of Life Scale (SCQOLS) among family caregivers of patients with advanced cancers in Singapore [2]. The SCQOLS consists of five domains and 51 items in total, namely Physical Well-being (12 items), Mental Well-being (10 items), Experience \& Meaning (12 items), Impact on Daily Life (13 items) and 
Financial Well-being (4 items). The items use a 5-point scale, from Not at All (0) to Very Much (4). Negatively worded items are recoded such that a higher score indicates a better QOL. The domain score is the mean of its item scores multiplied by 25 to scale to the $0-100$ scale, after applying the "half-rule" to handle item nonresponse (if any). That is, item non-responses are replaced by the mean of the observed item scores in the same domain if there are responses to at least half of the domain items. A weighted sum of the domain scores gives a total score, using the number of items in the domains divided by the total number of items as the weight. The validity and reliability of the SCQOLS and its domains have been demonstrated [2]. The questionnaire is available in the English and Chinese language (https://www.duke-nus.edu.sg/lcpc/resources/scqols-request-form). We had also demonstrated, using an equivalence study approach and having conditioned on demographic and health covariates, that the two language versions gave mean scores within an equivalence margin of $+/-0.5$ standard deviation [5].

The SCQOLS total and domain scores were scaled to range from 0 to 100 . However, the scale is not at the interval-ratio level of measurement. To facilitate interpretation of what is a high or low QOL score, reference values are needed. Furthermore, tabulation of the differences in QOL scores in relation to caregiver and patient characteristics will provide effect size benchmarks for the interpretation of intervention effects and differences between persons.

Reference values, also called "norms" or "standards", indicate the distribution of a measurement in a population. The concepts and methods for their development and clinical application are most elaborated in the assessment of fetal and child growth [6, 7]. Typically, a sample of "healthy" pregnancies/infants/children are recruited and the percentiles of an anthropometric measure in relation to personal characteristics (most commonly gestational age or age) are calculated. They are then used to "prescribe" what "normal" growth is, as opposed to using them to "describe" what the "observed distribution" is $[6,7]$. The scale of the data collection operation is often large. One reason is that a large number of people has to be screened in order to identify a "healthy" sample. Another reason is that the conventional way to estimate characteristic-specific (e.g. agespecific) percentiles is to group the observations into categories (e.g. age intervals) and then calculate the percentiles for each category separately. Therefore, each category will need a sufficiently large sample size $(\geq 200)$ [8]. This way of generating reference intervals ignores the fact that the observations from adjacent ordered categories are informative for estimating the percentiles in the flanked category. One response to this large sample size demand is to use broader categories, such that the multiplier for the sample size per category is smaller and therefore the total sample size required is more manageable [9]. This practice loses accuracy. For example, if 12-month age intervals are used, two children who are only 1 month apart may have different percentiles because they are at the upper and lower ends of adjacent age intervals, yet two other children who are 11 months apart may be shown to share the same percentiles because they are within the same age interval.

Modern research using regression or curve smoothing methods in the production of reference values substantially increase the level of precision or reduce the sample size requirements. Characteristics such as age are treated as continuous variables and pool all observations in the modelling $[6,7,10]$. Staffa et al. gave an introduction to the method in a medical context [11]. Cheung et al. applied this approach to the norming of cognitive function [9]. Using a regression-based approach for the estimation of reference intervals, a sample size of 70 has been considered the minimum acceptable sample size [10]. Furthermore, a sample size that exceeds 200 offers only minor incremental gain in precision [10]. Therefore, a sample size of at least 200 is a reasonable target. Furthermore, under this approach it is not mandatory to exclude "unhealthy" persons from the modelling. Percentiles can be obtained for both the healthy and unhealthy populations by including variables that differentiate them as predictors in the model that pools all observations. While the observations in the unhealthy population do not directly contribute information about the percentiles in the healthy population, they do contribute information about the effects of personal characteristics such as the age trend. It indirectly contributes to the accuracy in the percentile estimation for the healthy population. The potential of the regression approach does not seem to have been exploited in the construction of reference intervals for QOL measures.

We report our use of the quantile regression approach to the estimation of reference values for SCQOLS. Our primary aims are two-fold. First, we provide the regression equations to generate percentiles in relation to caregiver and patient characteristics. The equations can then be used to estimate the percentiles for a "mild" caregiver situation, similar to the "healthy" population in fetal and child growth, for prescription as the reference values to define what a poor or good QOL level is. Second, we provide the estimates of differences in median QOL scores between different levels of caregiver and patient characteristics. They serve as effect size benchmarks to facilitate the interpretation of the meaningfulness of a difference between persons or intervention groups. Our secondary aim is to demonstrate and 
discuss the use of a regression-based approach for constructing reference intervals in QOL research.

\section{Methods}

\section{Study setting and design}

Singapore is a multi-ethnic society. Chinese (74\%), Malay (13\%) and Indian (9\%) are the major ethnic groups; English is the lingua franca whereas bilingualism is common [12]. The National Cancer Centre is the largest public provider of outpatient cancer care in the country, serving $65 \%$ of the cancer patients in the public sector [13]. The patients who need inpatient care are usually admitted to the Singapore General Hospital.

Details of the study design and procedures have been previously published [2]. Briefly, family caregivers of patients with advanced cancers who were receiving care from the National Cancer Centre or Singapore General Hospital were recruited. Participants must be 21 years of age or older, able to communicate in either English or Chinese (Mandarin), aware of the patient's diagnosis, and the patients must have stage III or IV solid cancers. Consented caregivers were invited to self-administer the questionnaire in English or Chinese according to their own language preference. Some caregivers requested interviewer-administration. Informed consent was obtained before the survey. The Singapore Health Services Centralized Institutional Review Board approved this study.

The study comprised a baseline and a follow-up survey. The present manuscript only involves the baseline survey data, because the follow-up survey was only designed to estimate test-retest reliability. In addition to the SCQOLS and psychometric measures for assessment of the scale's validity, the baseline survey also included items on demographics, caregiving and health background.

\section{Measurements}

We consider four groups of predictor variables. (a) Caregiver demographics, including age, gender, ethnicity (Chinese, Malay, Indian and Others) and education (tertiary, secondary and primary or lower. (b) Survey procedures, including questionnaire language (English or Chinese) and mode of administration (self- or interviewer-administration). (c) A survey question that asked the caregivers whether s/he was "the only person", "the primary person" or "one of the few persons" who carries out caregiving duties for the patient. They were coded as 0 to 2, respectively; a lower value is anticipated to predict a worse QOL. For brevity, we refer to this variable as "caregiver role". (d) Patient characteristics, including performance status and cancer diagnosis. The performance status score ranged from 0 to 4 , representing "without symptoms", "with symptoms, fully ambulatory", "with symptoms, in bed less than 50\% during the day", "with symptoms, in bed more than 50\% during the day but not bedridden" and "bedridden", respectively [14].

\section{Statistical analysis}

Quantile regression was used to assess relationship between predictors and the 10th, 25th, 50th (median), 75th and 90th percentiles of each QOL score. By minimizing the sum of absolute deviation, as opposed to the least square regression that minimizes the sum of squared deviation, the quantile regression estimates the 50th percentile $[6,11]$. This unweighted estimation is also called the median regression. By allocating appropriate weights to the deviation above or below the fitted curve, the corresponding percentiles are estimated $[6,11]$.

Following Bonferroni adjustment for multiplicity (five percentiles), a predictor was kept in the final model if its regression coefficient showed $P<0.01$ in at least one of the five percentile equations in the initial multivariable model. Equivalently, this meant Bonferroni-adjusted P, or $\mathrm{P}[\mathrm{B}],<0.05$. For ethnicity (four categories) and education (three categories), if at least one of the indicator variables showed $P<0.01$ in the initial model, they were kept in the final model but categories that were not significantly different from each other (Wald test) would be combined. For levels of caregiver role and patient performance status (both ordinal variables), models treating them as categories or linear trends were both fitted. Since quantile regression is not based on maximum likelihood estimation, conventional methods for model selection are not available. For comparison of different models for caregiver role and patient performance status, we calculated the absolute deviations between the observed values and predicted median and compared the mean absolute deviation by paired-sample t-test. The simpler model (linear trend) was chosen if the difference was small and statistically non-significant. Since the analytic estimator of the standard error of quantile regression coefficients is known to be inaccurate, we used the bootstrap standard error with 100 replicates [6].

\section{Results}

\section{Descriptive summary}

Table 1 describes the study sample. A total of 612 caregivers were recruited. The mean age of the caregivers was 48 years; $61 \%$ were female; $85 \%$ were ethnic Chinese; $15 \%$ received primary education or below. About half of the participants chose to use the English questionnaire package; $90 \%$ self-administered the questionnaire. The percentage of caregivers who were the only, primary, and one of the few persons in the family who took care of the cancer patients were $21 \%, 35 \%$ and $44 \%$, respectively. Patient's performance status ranged from 0 (best) to 4 (worst). Colorectal (24\%), lung (21\%) and breast 
Table 1 Participant characteristics $(N=612)^{*}$

\begin{tabular}{ll}
\hline Characteristics & Mean (SD) \\
\hline Age (years) & $48(14)$ \\
Female & Gender \\
Male & $373(61.0 \%)$ \\
& $239(39.0 \%)$ \\
Chinese & Ethnicity \\
Malay & \\
Indian & $521(85.1 \%)$ \\
Others & $53(8.7 \%)$ \\
& $19(3.1 \%)$ \\
Tertiary & $19(3.1 \%)$ \\
Secondary & \\
Primary or below & \\
\hline
\end{tabular}

Questionnaire language

$\begin{array}{ll}\text { English } & 304(49.7 \%) \\ \text { Chinese } & 308(50.3 \%)\end{array}$

Mode of administration

Self

$551(90.0 \%)$

Interviewer

$61(10.0 \%)$

Caregiver role

0 Only person
1 Primary person
2 One of the few persons
Patient's performance status
0 (Best)
1
2
3
4 (Worst)

$126(20.6 \%)$

217 (35.5\%)

269 (44.0\%)

$71(11.6 \%)$

205 (33.5\%)

$81(13.2 \%)$

$170(27.8 \%)$

85 (13.9\%)

Patient's diagnosis

$\begin{array}{ll}\text { Colorectal } & 145(23.7 \%) \\ \text { Lung } & 129(21.1 \%) \\ \text { Breast } & 72(11.8 \%) \\ \text { Others } & 266(43.5 \%)\end{array}$

"Mean and standard deviation (SD) for continuous variables; frequency (N) and percent for categorical variables

${ }^{\text {T}}$ Percentages may not sum to 100 due to rounding
(12\%) cancers were the major diagnoses; all other diagnoses had frequencies smaller than $12 \%$ and were combined as "Others".

\section{QOL total score}

Table 2 shows the results of the initial quantile regression model. Age, gender, language version, mode of administration and cancer diagnosis had no association with any of the percentiles (each $P>0.01$ ). Ethnic Malay participants were 13 points higher than ethnic Chinese participants in the 10th percentile $(P<0.01$; significant after Bonferroni adjustment). Despite larger $P$ values, Indian and Others also had higher 10th percentile than the ethnic Chinese. Higher QOL total scores in the nonChinese ethnic groups were observed in most other percentiles. No statistically significant difference was found between Malay, Indian and Others in the five percentiles (each $P>0.10$ ). Ethnicity was regrouped as Chinese and non-Chinese in subsequent analysis of the total scores. Primary/below education was associated with lower 90th percentile $(P<0.01)$. No statistically significant difference was found between tertiary and secondary education in the five percentiles (each $P>0.10$ ). Education was dichotomized as secondary/above versus primary/below in subsequent analysis of the total scores.

For all five percentiles, caregiver roles and patient performance status showed a monotonic trend. The mean absolute deviation of the median regression model that kept caregiver role and patient performance status as categorical variables was 10.73 . Treating caregiver role and patient performance as linear trend variables according to their numeric codes gave mean absolute deviation of 10.78. Paired sample t-test showed $P=0.318$ for the difference. Hence the final model treated these two variables as quantitative variables with liner trends. The final model is presented in Table 3.

\section{Domain scores}

The final models for the five domains scores are presented in Tables 4, 5, 6, 7, 8. Similar to the prediction of the total score, caregiver role and patient performance status were predictors of all the domain scores except Experience \& Meaning.

For Physical Well-being, Malay and Others had higher but Indians had lower 10th percentile than ethnic Chinese participants (Table 4). Among the non-Chinese groups, there was statistically significant difference in the 10th percentile $(P<0.005)$ and non-statistically significant differences in the other percentiles (each $P>$ 0.10). Hence, we regrouped ethnicity to Chinese, Indian and Others (including Malay) for the percentile equations. Education was not associated with Physical Wellbeing. 
Table 2 Percentiles of QOL total score, initial model

\begin{tabular}{|c|c|c|c|c|c|c|c|c|c|c|}
\hline \multirow[t]{2}{*}{ Predictor } & 10th & percentile & 25 th & percentile & 50th & percentile & 75th & percentile & 90th & percentile \\
\hline & Coef. & $(95 \% \mathrm{Cl})$ & Coef. & $(95 \% \mathrm{Cl})$ & Coef. & $(95 \% \mathrm{Cl})$ & Coef. & $(95 \% \mathrm{Cl})$ & Coef. & $(95 \% \mathrm{Cl})$ \\
\hline \multicolumn{11}{|l|}{ Age } \\
\hline (per 10 years) & 1.3 & $(-0.9,3.6)$ & 1.3 & $(-0.5,3.1)$ & 1.1 & $(-0.1,2.5)$ & 1.7 & $(0.5,2.8)$ & 0.8 & $(-0.5,2.1)$ \\
\hline \multicolumn{11}{|l|}{ Gender } \\
\hline Female & 0 & & 0 & & 0 & & 0 & & 0 & \\
\hline Male & 1.4 & $(-4.8,7.7)$ & 1.2 & $(-2.6,4.9)$ & -0.4 & $(-3.5,2.6)$ & -1.5 & $(-4.0,1.1)$ & -2.1 & $(-4.8,0.7)$ \\
\hline \multicolumn{11}{|l|}{ Ethnicity } \\
\hline Chinese & 0 & & 0 & & 0 & & 0 & & 0 & \\
\hline Malay & $12.7^{*}$ & $(4.0,21.4)$ & 5.8 & $(-2.8,14.5)$ & 4.1 & $(-1.1,9.3)$ & 7.1 & $(0.3,14.0)$ & 4.5 & $(-1.1,10.0)$ \\
\hline Indian & 13.6 & $(1.3,25.9)$ & 5.0 & $(-5.3,15.3)$ & -0.9 & $(-8.9,7.1)$ & 3.2 & $(-6.1,12.5)$ & 0.1 & $(-6.5,6.8)$ \\
\hline Others & 15.7 & $(3.0,21.4)$ & $14.7^{*}$ & $(4.5,25.0)$ & 8.4 & $(0.5,16.4)$ & 4.7 & $(-1.8,11.3)$ & 0.2 & $(-6.4,6.9)$ \\
\hline \multicolumn{11}{|l|}{ Education } \\
\hline Tertiary & 0 & & 0 & & 0 & & 0 & & 0 & \\
\hline Secondary & -4.0 & $(-9.7,1.6)$ & -1.0 & $(-6.7,4.8)$ & 1.9 & $(-1.8,5.5)$ & 0.3 & $(-2.7,3.3)$ & 0.7 & $(-2.5,3.9)$ \\
\hline Primary/below & -1.8 & $(-9.2,5.7)$ & -3.9 & $(-10.0,2.1)$ & -3.0 & $(-7.7,1.7)$ & -5.5 & $(-10.2,-0.9)$ & $-6.3^{*}$ & $(-9.9,-2.7)$ \\
\hline \multicolumn{11}{|l|}{ Language } \\
\hline English & 0 & & 0 & & 0 & & 0 & & 0 & \\
\hline Chinese & 5.5 & $(0.3,10.7)$ & 3.2 & $(-7.8,6.3)$ & 1.2 & $(-2.2,4.6)$ & 1.3 & $(-2.0,4.5)$ & -0.4 & $(-4.0,3.3)$ \\
\hline \multicolumn{11}{|c|}{ Mode of administration } \\
\hline Self & 0 & & 0 & & 0 & & 0 & & 0 & \\
\hline Interviewer & -1.2 & $(-11.6,9.2)$ & -0.8 & $(-7.8,6.3)$ & -2.1 & $(-7.7,3.4)$ & -2.3 & $(-8.2,3.7)$ & 1.9 & $(-3.8,7.6)$ \\
\hline \multicolumn{11}{|l|}{ Caregiver role } \\
\hline 0 & 0 & & 0 & & 0 & & 0 & & 0 & \\
\hline 1 & 4.5 & $(-1.7,10.7)$ & $8.8^{*}$ & $(2.8,14.8)$ & 5.8 & $(0.9,10.7)$ & 4.3 & $(0.2,8.3)$ & 1.8 & $(-2.7,6.3)$ \\
\hline 2 & $12.5^{*}$ & $(6.3,18.8)$ & $15.1^{*}$ & $(10.2,20.0)$ & $9.8^{*}$ & $(4.3,15.3)$ & $7.3^{*}$ & $(3.4,11.2)$ & 3.6 & $(-0.7,7.9)$ \\
\hline \multicolumn{11}{|l|}{ Performance status } \\
\hline 0 & 0 & & 0 & & 0 & & 0 & & 0 & \\
\hline 1 & -5.5 & $(-16.7,5.8)$ & $-6.8^{*}$ & $(-11.9,-1.8)$ & -4.2 & $(-8.0,-0.3)$ & -3.1 & $(-6.3,0.0)$ & -2.0 & $(-5.6,1.6)$ \\
\hline 2 & -14.9 & $(-27.7,-2.1)$ & $-14.4^{*}$ & $(-21.3,-7.4)$ & $-8.0^{*}$ & $(-13.6,-2.4)$ & -5.9 & $(-11.0,-0.7)$ & -4.4 & $(-8.9,0.2)$ \\
\hline 3 & $-16.3^{*}$ & $(-28.0,-4.6)$ & $-14.7^{*}$ & $(-20.1,-9.2)$ & $-9.7^{*}$ & $(-13.7,-5.6)$ & $-7.4^{*}$ & $(-11.1,-3.8)$ & $-5.7^{*}$ & $(-9.4,-1.9)$ \\
\hline 4 & $-20.4^{*}$ & $(-32.3,-8.5)$ & $-22.2^{*}$ & $(-30.8,-13.6)$ & $-14.5^{*}$ & $(-20.6,-8.5)$ & $-8.9^{*}$ & $(-13.4,-4.3)$ & $-8.8^{*}$ & $(-13.8,-3.7)$ \\
\hline \multicolumn{11}{|l|}{ Diagnosis } \\
\hline Colorectal & 0 & & 0 & & 0 & & 0 & & 0 & \\
\hline Breast & 5.0 & $(-5.2,15.3)$ & 0.3 & $(-6.6,7.1)$ & 1.1 & $(-4.8,7.1)$ & 0.3 & $(-4.3,5.0)$ & 3.6 & $(-2.1,9.3)$ \\
\hline Lung & 8.6 & $(-0.1,17.2)$ & 2.2 & $(-3.3,7.7)$ & 0.8 & $(-3.4,4.9)$ & -0.3 & $(-4.5,3.9)$ & 2.1 & $(-1.9,6.2)$ \\
\hline Others & 6.3 & $(-1.6,14.3)$ & 4.1 & $(-0.8,9.1)$ & 1.2 & $(-2.0,4.4)$ & -1.0 & $(-3.7,1.8)$ & -0.3 & $(-3.6,3.1)$ \\
\hline Intercept & $36.4^{*}$ & $(15.3,57.5)$ & $47.2^{*}$ & $(5.3,59.1)$ & $61.3^{*}$ & $(51.4,71.1)$ & $65.9^{*}$ & $(55.4,76.5)$ & $76.5^{*}$ & $(66.3,86.6)$ \\
\hline
\end{tabular}

${ }^{\bar{P} P}<0.01$

For Mental Well-being, primary/below education was associated with a lower median (Table 5). There was no significant difference between tertiary and secondary education $(P>0.10)$ and hence they were combined.

For Experience \& Meaning, none of the predictors were associated with the scores except ethnicity (Table 6). All three non-Chinese groups had higher scores than the
Chinese. There was no significant difference among the three non-Chinese groups in any of the five percentiles (each $P>0.10$ ) and hence they were combined.

Only caregiver role and patient performance status were related to Impact on Daily Life (Table 7).

Age was related only to the Financial Well-being score (Table 8). Caregivers with tertiary, secondary and primary/ 
Table 3 Percentiles of QOL total score, final model

\begin{tabular}{|c|c|c|c|c|c|c|c|c|c|c|}
\hline Predictor & $\begin{array}{l}\text { 10th } \\
\text { Coef. }\end{array}$ & $\begin{array}{l}\text { percentile } \\
(95 \% \mathrm{Cl})\end{array}$ & $\begin{array}{l}\text { 25th } \\
\text { Coef. }\end{array}$ & $\begin{array}{l}\text { percentile } \\
(95 \% \text { Cl) }\end{array}$ & $\begin{array}{l}\text { 50th } \\
\text { Coef. }\end{array}$ & $\begin{array}{l}\text { percentile } \\
(95 \% \mathrm{Cl})\end{array}$ & $\begin{array}{l}\text { 75th } \\
\text { Coef. }\end{array}$ & $\begin{array}{l}\text { percentile } \\
(95 \% \mathrm{Cl})\end{array}$ & $\begin{array}{l}\text { 90th } \\
\text { Coef. }\end{array}$ & $\begin{array}{l}\text { percentile } \\
(95 \% \mathrm{Cl})\end{array}$ \\
\hline \multicolumn{11}{|l|}{ Ethnicity } \\
\hline Chinese & 0 & & 0 & & 0 & & 0 & & 0 & \\
\hline Others & 7.3 & $(0.6,14.0)$ & 6.1 & $(0.8,11.5)$ & 3.0 & $(0.2,5.9)$ & 3.3 & $(-1.5,8.1)$ & 3.9 & $(0.6,7.1)$ \\
\hline \multicolumn{11}{|l|}{ Education } \\
\hline Secondary/above & 0 & & 0 & & 0 & & 0 & & 0 & \\
\hline Primary/below & 2.5 & $(-3.1,8.0)$ & -1.1 & $(-6.7,4.5)$ & -3.2 & $(-6.6,0.2)$ & $-4.7^{*}$ & $(-8.0,-1.4)$ & -5.1 & $(-9.1,-1.0)$ \\
\hline Caregiver role & $5.7^{*}$ & $(2.2,9.3)$ & $6.4^{*}$ & $(4.0,8.7)$ & $3.1^{*}$ & $(1.0,5.2)$ & $1.8^{*}$ & $(0.7,3.0)$ & 0.6 & $(-0.9,2.2)$ \\
\hline Performance status & $-5.1^{*}$ & $(-7.7,-2.6)$ & $-4.7^{*}$ & $(-6.4,-2.9)$ & $-3.3^{*}$ & $(4.6,-2.2)$ & $-2.7^{*}$ & $(-3.3,-2.1)$ & $-2.2^{*}$ & $(-3.1,-1.3)$ \\
\hline Intercept & $52.0^{*}$ & $(42.6,61.5)$ & $61.1^{*}$ & $(56.9,65.2)$ & $73.4^{*}$ & $(69.1,77.6)$ & $82.4^{*}$ & $(80.1,84.6)$ & $88.3^{*}$ & $(85.1,91.4)$ \\
\hline
\end{tabular}

${ }^{*} P<0.01$

${ }^{\dagger}$ Example: The 10th percentile of an ethnic Indian caregiver with tertiary education who is the only family member (caregiver role $=0$ ) giving care to a patient who has symptoms but is ambulatory (performance status $=1)$ is $52.0+7.3+0+(0 \times 5.7)+(1 \times[-5.1])=54.2$

below education background showed a gradient in this domain. Furthermore, as previously reported [2], the Financial Well-being score showed a heavy ceiling effect (24.7\%). Hence, the quantile regression equation for the 90th percentile had intercept 100 and the regression coefficients for the predictors were all zero.

\section{Percentiles in a "mild" state}

We present the predicted QOL total score percentiles for caregivers who were ethnic Chinese (mode) with tertiary education (mode), who were the primary (but not the only) caregivers in the families (caregiver role $=1$ ) and whose care-recipients had disease symptoms but were fully ambulatory (performance status $=1$ ) as a "typical" caregiver in a "mild" state, analogous to the "healthy" sample in fetal and child growth references. According to Table 3, the predicted 10th percentile for this group of caregivers is

$$
10^{\text {th }} \text { percentile }=52.0+0+0+(1 \times 5.7)+(1 \times[-5.1])=52.6 .
$$

Following the same application of the equations in Table 3, the predicted 10th, 25th, 50th, 75th and 90th percentiles (standard error of prediction, not shown in Tables) of the "typical" caregivers in the "mild" state $(N=55)$ were $52.6(2.5), 62.8$ (1.3), $73.2(1.0)$, 81.5 (0.7) and 86.7 (0.9), respectively (Fig. 1).

To compare the gain in precision by using quantile regression versus the conventional approach, we contrast the results for the caregivers above versus the percentiles estimated from the same group without using regression analysis. The percentiles (standard error) observed in this group of caregivers $(N=55)$ were 47.4 (5.6), 64.2 (2.3), 74.0 (2.5), 81.1 (2.7) and 89.6 (2.5), respectively. While the percentile estimates obtained from the two approaches were similar (except the 10th percentile), the standard errors were much smaller in the quantile regression approach.

Finally, based on the equations in Tables $3,4,5,6,7$, 8 , the percentiles of the QOL domain and total scores of caregivers in the "mild" state and, where applicable, in the most common demographic category (ethnic Chinese and tertiary education) and at mean age ( 48 years) are shown in Fig. 1.

Table 4 Percentiles of QOL Physical Well-being score, final model

\begin{tabular}{|c|c|c|c|c|c|c|c|c|c|c|}
\hline Predictor & $\begin{array}{l}\text { 10th } \\
\text { Coef. }\end{array}$ & $\begin{array}{l}\text { percentile } \\
(95 \% \mathrm{Cl})\end{array}$ & $\begin{array}{l}25 \text { th } \\
\text { Coef. }\end{array}$ & $\begin{array}{l}\text { percentile } \\
(95 \% \mathrm{Cl})\end{array}$ & $\begin{array}{l}\text { 50th } \\
\text { Coef. }\end{array}$ & $\begin{array}{l}\text { percentile } \\
(95 \% \mathrm{Cl})\end{array}$ & $\begin{array}{l}\text { 75th } \\
\text { Coef. }\end{array}$ & $\begin{array}{l}\text { percentile } \\
(95 \% \mathrm{Cl})\end{array}$ & $\begin{array}{l}\text { 90th } \\
\text { Coef. }\end{array}$ & $\begin{array}{l}\text { percentile } \\
(95 \% \mathrm{Cl})\end{array}$ \\
\hline \multicolumn{11}{|l|}{ Ethnicity } \\
\hline Chinese & 0 & & 0 & & 0 & & 0 & & 0 & \\
\hline Indian & -9.7 & $(-25.1,5.7)$ & -9.4 & $(-30.9,12.1)$ & -4.2 & $(-18.9,10.6)$ & 0.0 & $(-8.4,8.4)$ & 1.0 & $(-6.3,8.4)$ \\
\hline Others & $14.6^{*}$ & $(7.4,21.7)$ & 7.8 & $(1.7,3.9)$ & $6.3^{*}$ & $(2.2,10.3)$ & 4.2 & $(0.9,7.4)$ & 2.1 & $(-0.4,4.6)$ \\
\hline Caregiver role & $13.2^{*}$ & $(8.6,17.8)$ & $10.4^{*}$ & $(7.0,13.8)$ & $5.6^{*}$ & $(3.0,8.1)$ & $4.2^{*}$ & $(2.4,5.9)$ & 1.0 & $(-0.6,2.6)$ \\
\hline Performance status & $-4.9^{*}$ & $(-7.8,-1.9)$ & $-5.6^{*}$ & $(-7.9,-3.6)$ & $-5.6^{*}$ & $(-6.8,-4.3)$ & $-4.1^{*}$ & $(-5.3,-3.1)$ & $-2.1^{*}$ & $(-2.9,1.3)$ \\
\hline Intercept & $41.7^{*}$ & $(30.4,52.9)$ & $62.5^{*}$ & $(55.1,69.9)$ & $81.9^{*}$ & $(76.6,87.3)$ & $91.7^{*}$ & $(87.5,95.8)$ & $97.9^{*}$ & $(94.9,100.9)$ \\
\hline
\end{tabular}

${ }^{*} P<0.01$

${ }^{\dagger}$ Example: The 10th percentile of an Indian caregiver who is the only family member (caregiver role $=0$ ) giving care to a patient who has symptoms but is ambulatory (performance status $=1)$ is $41.7-9.7+(0 \times 13.2)+(1 \times[-4.9])=27.1$ 
Table 5 Percentiles of QOL Mental Well-being score, final model

\begin{tabular}{|c|c|c|c|c|c|c|c|c|c|c|}
\hline \multirow[t]{2}{*}{ Predictor } & 10th & percentile & 25 th & percentile & 50th & percentile & 75th & percentile & 90th & percentile \\
\hline & Coef. & $(95 \% \mathrm{Cl})$ & Coef. & $(95 \% \mathrm{Cl})$ & Coef. & $(95 \%$ Cl) & Coef. & $(95 \%$ Cl) & Coef. & $(95 \%$ Cl) \\
\hline \multicolumn{11}{|l|}{ Education } \\
\hline Secondary/above & 0 & & 0 & & 0 & & 0 & & 0 & \\
\hline Primary/below & -10.6 & $(-20.8,-0.5)$ & -10.0 & $(-18.1,-1.9)$ & $-10.8^{*}$ & $(-18.3,-3.4)$ & -6.7 & $(-13.9,0.6)$ & 0.6 & $(-5.7,6.9)$ \\
\hline Caregiver role & $6.9^{*}$ & $(2.2,11.6)$ & 1.6 & $(-0.6,5.6)$ & 3.3 & $(0.2,6.4)$ & 2.5 & $(-0.4,5.4)$ & 2.5 & $(-0.8,5.8)$ \\
\hline Performance status & $-4.4^{*}$ & $(-7.1,-1.7)$ & $-5.0^{*}$ & $(-7.0,-3.0)$ & $-3.3^{*}$ & $(-4.9,-1.8)$ & $-3.3^{*}$ & $(-5.2,-1.5)$ & $-3.1^{*}$ & $(-4.7,-1.6)$ \\
\hline Intercept & $35.0^{*}$ & $(26.5,43.5)$ & $55.0^{*}$ & $(47.5,62.5)$ & $65.8^{*}$ & $(60.5,71.2)$ & $78.3^{*}$ & $(71.3,85.4)$ & $86.9^{*}$ & $(80.7,93.0)$ \\
\hline
\end{tabular}

${ }^{*} P<0.01$

${ }^{\dagger}$ Example: The 10th percentile of a caregiver who has tertiary education background and is the only family member (caregiver role $=0$ ) giving care to a patient who has symptoms but is ambulatory (performance status $=1)$ is $35.0+0+(0 \times 6.9)+(1 \times[-4.4])=30.6$

\section{Discussion}

With differences in socio-cultural context between the East and West and the sub-optimal validity of some caregiver QOL measurement scales for use in Asia [3, $4]$, the recent development of the SCQOLS is a valuable addition. Clinicians often need to classify test results in order to differentiate care recipients who require different types or level of services. In particular, it is important to identify care recipients who have an "abnormal" level of health indicators so as to provide timely interventions. Researchers may also have similar needs, such as to define inclusion/exclusion criteria for study enrolment and to define caseness endpoints. The percentiles we have presented can be used by clinicians and researchers of family caregivers to serve these purposes.

The use of regression analysis to estimate reference values tends to be more precise than the conventional way of separate calculation for each covariate interval or covariate combination. As we have seen in the illustration, the conventional way of estimating percentiles has substantially larger standard errors. The quantile regression is a precise and powerful tool for this purpose. Relative to other modern methods used in the construction of fetal and child growth references, quantile regression has the advantages of not involving a normal distribution assumption and ease of inclusion of multiple predictors.

The study sample's demographic characteristics, such as education, age and gender, are similar to the caregiver profiles shown in other surveys of caregivers [15] and caregivers of cancer patients in Singapore [16]. As such, we believe the sample is representative of the target population.

We have previously provided evidence that the English and Chinese versions of the SCQOLS achieved equivalence in mean total and domain scores [5]. In the present analysis, we found no evidence of difference between the two language versions in any of the five percentiles in the SCQOLS total or domain scores. The availability of two language versions that have similar properties makes the SCQOLS suitable for use not only in Singapore but also in other countries where one or both of these languages are commonly used and in cross-country comparison.

Two surveys of the Singapore general population that used the Short Form 36 Health Survey showed that, having adjusted for covariates, ethnic Indian persons tended to score lower than other ethnic groups on the Physical Component Summary, but not the Mental Component Summary $[17,18]$. While studies of general population do not directly shed lights on the caregiver population, they appear to be consistent with the present finding of ethnic Indian caregivers reporting lower level of Physical Well-being.

The Experience \& Meaning domain captures strengths and sense making that caregivers may experience despite adversity [2]. We found that ethnic Chinese tended to score lower than the other ethnic groups in Singapore.

Table 6 Percentiles of QOL Experience \& Meaning score, final model

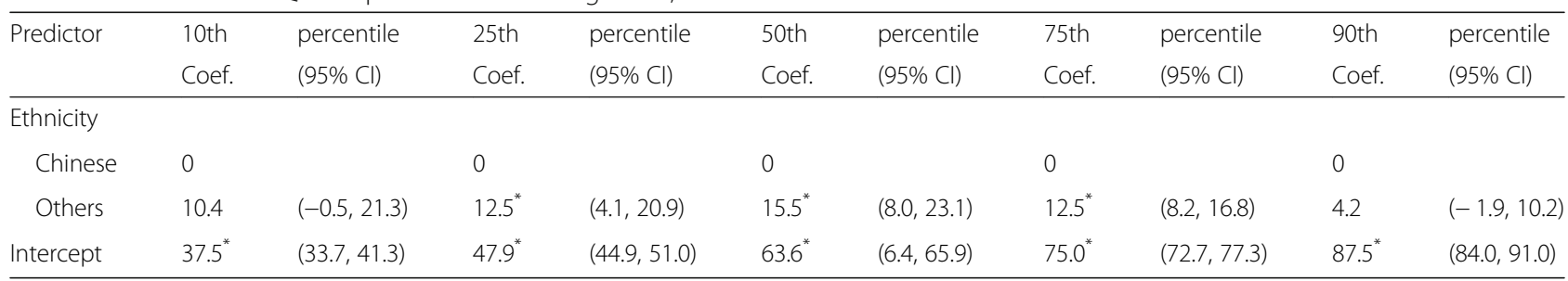

${ }^{*} P<0.01$

${ }^{\dagger}$ Example: The 10th percentile of a Chinese or Other caregiver is 37.5 or $37.5+10.4=47.9$, respectively 
Table 7 Percentiles of QOL Impact on Daily Life score, final model

\begin{tabular}{|c|c|c|c|c|c|c|c|c|c|c|}
\hline Predictor & 10th & percentile & 25th & percentile & 50th & percentile & 75th & percentile & 90th & percentile \\
\hline & Coef. & $(95 \% \mathrm{Cl})$ & Coef. & $(95 \% \mathrm{Cl})$ & Coef. & $(95 \% \mathrm{Cl})$ & Coef. & $(95 \% \mathrm{Cl})$ & Coef. & $(95 \% \mathrm{Cl})$ \\
\hline Caregiver role & $8.8^{*}$ & $(4.6,13.1)$ & $7.1^{*}$ & $(2.9,11.2)$ & $3.7^{*}$ & $(1.0,6.4)$ & 2.3 & $(0.4,4.3)$ & 1.0 & $(-0.7,2.1)$ \\
\hline Performance status & $-6.5^{*}$ & $(-9.6,-3.5)$ & $-7.1^{*}$ & $(-9.6,-4.5)$ & $-4.4^{*}$ & $(-5.7,-3.2)$ & $-2.7^{*}$ & $(-3.7,-1.7)$ & $-1.8^{*}$ & $(-2.6,-1.0)$ \\
\hline Intercept & $46.2^{*}$ & $(36.6,55.7)$ & $67.9^{*}$ & $(60.2,75.7)$ & $84.6^{*}$ & $(80.3,88.9)$ & $92.7^{*}$ & $(89.8,95.5)$ & $98.0^{*}$ & $(95.7,100.2)$ \\
\hline
\end{tabular}

${ }^{*} P<0.01$

${ }^{\dagger}$ Example: The 10th percentile of a caregiver who is the only family member (caregiver role $=0$ ) giving care to a patient who has symptoms but is ambulatory (performance status $=1)$ is $46.2+(0 \times 8.8)+(1 \times[-6.5])=39.7$

A study of the caregivers of community-dwelling Singaporeans aged 75 years or above showed that ethnic Malay and Indian caregivers had higher level of Caregiver Esteem [19]. This may be a contributor to the observed difference in the Experience \& Meaning domain.

A limitation of the present study is the relatively small number of non-Chinese caregivers. The estimates for the differences between ethnic groups should be considered tentative and will need further research to calibrate.

In the fetal and child growth context, samples are often limited to "healthy" population such that the percentiles derived are prescriptive instead of descriptive. For this reason, they are sometimes call growth "standards" instead of "reference values" [6]. Due to the variation in inclusion/exclusion criteria to define a healthy population, percentiles offered by a study may not fully satisfy the requirements of all users. The advantage of our approach to norming QOL is that the users can define a "mild" state flexibly. In our illustration, we focus on defining a "mild" state as (a) the care recipients being ambulatory despite having disease symptoms (performance status $=1$ ) and $(b)$ the caregiver being the primary but not the only person in the family to carry out caregiving duties (caregiver role $=1$ ). The percentiles can be considered the QOL standards in a realistically favourable situation. While it is possible to select the best scenario (performance status $=0$ and caregiver role $=2$ ) to calculate the percentiles, it may not be realistic. Nevertheless, users of the SCQOLS and the quantile regression equations may do so if they consider it appropriate for their specific purposes.

We did not plan the study to define the minimal clinically important difference, the estimation of which usually requires longitudinal data with a sufficient amount of follow-up time to allow for changes in QOL to occur [20]. This is another limitation of our study. Nevertheless, the quantile regression results show us the differences in median QOL scores between important variables such as patient performance status and caregiver role. These two variables were predictive of most of the SCQOLS scores. Despite variations between domains, their adjacent categories mainly differed by about 3 to 4 points in the median scores. These differences can serve as benchmarks to help with the interpretation of whether an observed difference between persons or between intervention groups are practically meaningful.

Table 8 Percentiles of QOL Financial Well-being score, final model

\begin{tabular}{|c|c|c|c|c|c|c|c|c|c|c|}
\hline \multirow[t]{2}{*}{ Predictor } & 10th & percentile & 25th & percentile & 50th & percentile & 75th & percentile & 90th & percentile \\
\hline & Coef. & $(95 \% \mathrm{Cl})$ & Coef. & $(95 \% \mathrm{Cl})$ & Coef. & (95\% Cl) & Coef. & (95\% Cl) & Coef. & $(95 \%$ Cl) \\
\hline \multicolumn{11}{|l|}{ Age } \\
\hline (per 10 years) & 2.3 & $(-3.5,8.0)$ & 6.0 & $(0.6,11.3)$ & $5.5^{*}$ & $(2.9,8.2)$ & 2.2 & $(-0.1,4.6)$ & 0 & $(0,0)$ \\
\hline \multicolumn{11}{|l|}{ Education } \\
\hline Tertiary & 0 & & 0 & & 0 & & 0 & & 0 & \\
\hline Secondary & -12.2 & $(-27.4,3.0)$ & $-16.4^{*}$ & $(-28.8,-4.0)$ & -6.8 & $(-14.5,0.8)$ & -1.7 & $(-4.7,1.3)$ & 0 & $(0,0)$ \\
\hline Primary/below & -15.8 & $(-35.0,3.4)$ & $-27.1^{*}$ & $(-42.7,-11.5)$ & $-22.3^{*}$ & $(-33.0,-11.5)$ & $-14.7^{*}$ & $(-26.0,-3.5)$ & 0 & $(0,0)$ \\
\hline Caregiver role & 9.0 & $(0.8,17.3)$ & $12.8^{*}$ & $(5.4,20.2)$ & $13.5^{*}$ & $(8.7,18.2)$ & $5.9^{*}$ & $(2.2,9.6)$ & 0 & $(0,0)$ \\
\hline Performance status & -5.3 & $(-11.6,1.0)$ & -2.8 & $(-7.2,1.4)$ & -2.5 & $(-4.7,-0.2)$ & -0.6 & $(-1.9,0.7)$ & 0 & $(0,0)$ \\
\hline Intercept & 20.6 & $(-14.2,55.3)$ & 21.1 & $(-10.4,52.7)$ & $42.3^{*}$ & $(25.6,59.1)$ & $78 . .2^{*}$ & $(63.2,93.1)$ & 100.0 & $(100,100)$ \\
\hline
\end{tabular}

${ }^{*} P<0.01$

${ }^{\dagger}$ Example: The 10th percentile of a 50 -year old caregiver who has tertiary education and is the only family member (caregiver role $=0$ ) giving care to a patient who has symptoms but is ambulatory (performance status $=1$ ) is $20.6+(5 \times 2.3)+0+(0 \times 9.0)+(1 \times[-5.3])=26.8$ 


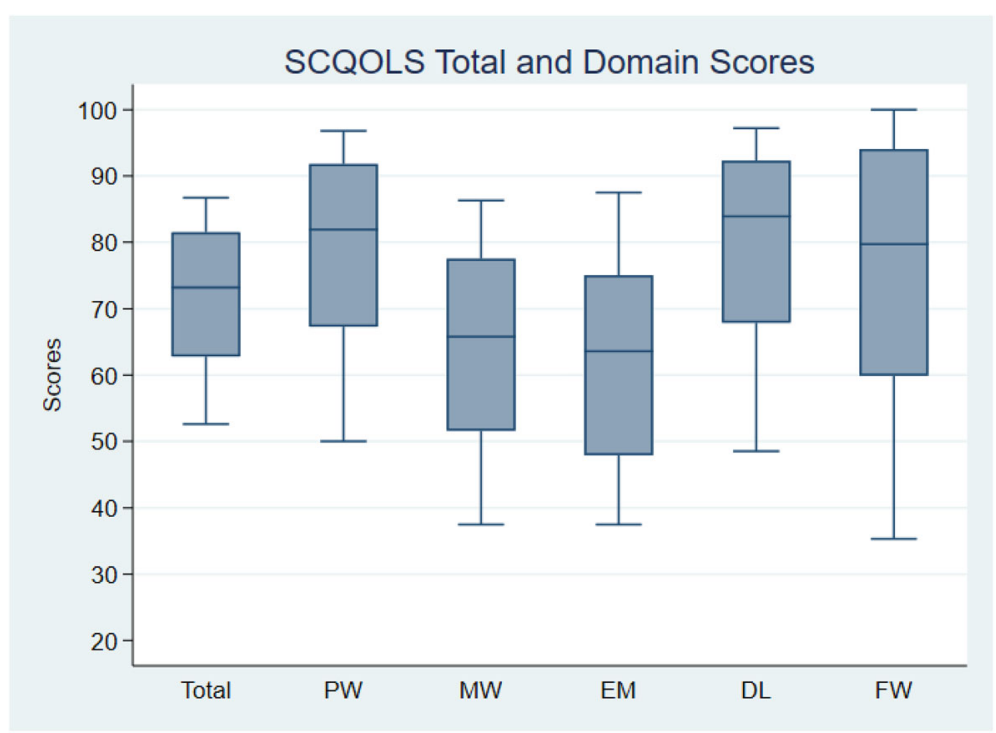

Fig. 1 Plots of 10th, 25th, 50th, 75th and 90th percentiles of quality of life scores of caregivers in a "mild" state (patient performance status =1 and caregiver role $=1$ ) and, where applicable, in the most common demographic category (ethnic Chinese and tertiary education) and at mean age (48 years) in the survey. Calculation based on equations in Tables 3, 4, 5, 6, 7, 8. PW: Physical Well-being; MW: Mental Well-being; EM: Experience \& Meaning; DL: Impact on Daily Life; FW: Financial Well-being

\section{Conclusions}

Percentiles and effect size benchmarks are available for the SCQOLS. They will facilitate the assessment of family caregivers in an Asian context.

\section{Abbreviations}

QOL: Quality of Life; SCQOLS: Singapore Caregiver Quality of Life Scale;

SD: Standard deviation

\section{Acknowledgements}

Not applicable.

\section{Authors' contributions}

YBC, SHSN, GMY, IT, GLL, and HLW conceived and designed the study. YBC, SHSN, GMY, IT, GLL and HLW developed the draft version of the measurement scale. SHSN, IT, GMY, JT, ARXK and DLMQ planned the data collection procedures and implemented the data collection. YBC performed statistical analysis. YBC drafted the first version of the manuscript. All authors participated in critical review and revision of the manuscript and approve the submission.

\section{Funding}

This study was funded by the National Medical Research Council, Singapore (NMRC/HSRG/0057/2016) and the Lien Centre for Palliative Care (LCPC-IN160004). Grace Yang received salary support from the Lien Centre for Palliative

\section{Availability of data and materials}

The de-identified dataset analysed is available from ScholarBank@NUS (https://doi.org/10.25540/AXN8-5EKD).

\section{Ethics approval and consent to participate}

All procedures performed in studies involving human participants were in accordance with the ethical standards of the institutional and/or national research committee (Centralized Institutional Review Board of the Singapore Health Services \#2016/2243) and with the 1964 Helsinki declaration and its later amendments or comparable ethical standards.

Informed consent was obtained from all individual participants included in the study.

\section{Consent for publication}

Not applicable.

\section{Competing interests}

All authors declare that they have no potential conflict of interest.

\section{Author details}

${ }^{1}$ Program in Health Services \& Systems Research, Duke-NUS Medical School, 20 College Road, Singapore 169856, Singapore. ${ }^{2}$ Centre for Quantitative Medicine, Duke-NUS Medical School, Level 6, Academia, 20 College Road, Singapore 169856 , Singapore. ${ }^{3}$ Centre for Child Health Research, Tampere University, Arvo Ylpönkatu 34, 33520 Tampere, Finland. ${ }^{4}$ Division of Supportive and Palliative Care, National Cancer Centre Singapore, 11 Hospital Drive, Singapore 169610, Singapore. ${ }^{5}$ Lien Centre for Palliative Care, Duke-NUS Medical School, 20 College Road, Singapore 169856, Singapore. ${ }^{6}$ Department of Social Work, National University of Singapore, 3 Arts Link, Singapore 117570 , Singapore. ${ }^{7}$ Department of Rheumatology and Immunology, Singapore General Hospital, Outram road, Singapore 169608, Singapore. ${ }^{8}$ Saw Swee Hock School of Public Health, National University of Singapore (NUS), National University Health System (NUHS), 12 Science Drive 2, Singapore 117549, Singapore. ${ }^{9}$ Department of Pharmacy, Faculty of Science, National University of Singapore, 18 Science Drive 4, Singapore 117559, Singapore.

Received: 26 November 2019 Accepted: 23 April 2020

Published online: 06 May 2020

\section{References}

1. Albers, G., Echteld, M. A., de Vet, H. C., et al. (2010). Evaluation of quality-oflife measures for use in palliative care: A systematic review. Palliative Medicine, 24, 17-37.

2. Cheung, Y. B., Neo, S. H. S., Teo, I., et al. (2019). Development and evaluation of a quality of life measurement scale in English and Chinese of family caregivers of patients with advanced cancers. Health and Quality of Life Outcomes, 17(1), 35 .

3. Lee, G. L., Ow, M. Y. L., Akhileswaran, R., et al. (2015). Quality of life domains important and relevant to family caregivers of advanced cancer patients in an Asian population - a qualitative study. Quality of Life Research, 24(4), 817828.

4. Duan, J., Fu, J., Gao, H., et al. (2015). Factor analysis of the caregiver quality of life index-Cancer (CQOLC) scale for Chinese cancer caregivers: A 
preliminary reliability and validity study of the CQOLC-Chinese version. PLOS One, 10(2), e0116438.

5. Cheung, Y. B., Yang, G. M., Neo, S. H. S., et al. (2018). A locally developed quality of life measurement scale for family caregivers of patients with advanced cancers in Singapore. Value in Health, 21(Suppl 2), S100.

6. Cheung, Y. B. (2014). Statistical analysis of human growth and development. Boca Raton: CRC Press.

7. WHO. (2006). WHO child growth standards: Length/height-for-age, weight-forage, weight-for-length, weight-for-height and body mass index-for-age: Methods and development. Geneva: WHO.

8. WHO Expert Committee. (1995). Physical status: The use and interpretation of anthropometry. Geneva: WHO.

9. Cheung, Y. B., Xu, Y., Feng, L., et al. (2015). Unconditional and conditional standards using cognitive function curves for the modified mini-mental state examination: Cross-sectional and longitudinal analyses in older Chinese adults in Singapore. American Journal of Geriatric Psychiatry, 23, 915-924.

10. Virtanen, A., Kairisto, V., \& Uusipaikka, E. (1998). Regression-based reference limits: Determination of sufficient sample size. Clinical Chemistry, 44(11), 2353-2358.

11. Staffa, S. J., KOhane, D. S., \& Zurakowski, D. (2019). Quantile regression and its applications: A primer for anesthesiologists. Anesthesia and Analgesia, 128(4), 820-830.

12. Department of Statistics. (2015). General household survey 2015. Singapore: Department of Statistics.

13. National Cancer Center Singapore. (2016). 2016 fact book. Singapore: National Cancer Center Singapore.

14. Lee, C. F., Ng, R., Luo, N., \& Cheung, Y. B. (2014). Patient-reported outcomes are associated with patient-oncologist agreement of performance status in a multi-ethnic Asian population. Supportive Care in Cancer, 22(12), 32013208.

15. Zheng, Z. (2011). Caregiving in Singapore. Statistics Singapore Newsletter, September (Vol. 2011, pp. 12-14),

16. Lim, H. A., Tan, J. Y., Chua, J., et al. (2017). Quality of life of family caregivers of cancer patients in Singapore and globally. Singapore Medical Journal, 58(5), 258-261.

17. Thumboo, J., Fong, K. Y., Machin, D., et al. (2003). Quality of life in an urban Asian population: The impact of ethnicity and socio-economic status. Social Science and Medicine, 56(8), 1761-1772.

18. Leow, M. K. S., Griva, K., Choo, R., et al. (2013). Determinants of health-related quality of life (HRQoL) in the multi-ethnic Singapore population - A national cohort study. PLoS One, 8(6), e67138.

19. Ostbye, T., Malhotra, R., Malhotra, C., Arambepola, C., \& Chan, A. (2013). Does support from foreign domestic workers decrease the negative impact of informal caregiver? Result from Singapore survey on informal caregiving. Journal of Gerontology, Series B, 68(4), 609-621.

20. Fayers, P., \& Machin, D. (2016). Quality of Life: The Assessment, Analysis and Reporting of Patient-Reported Outcomes (3rd ed.). Chichester: Wiley.

\section{Publisher's Note}

Springer Nature remains neutral with regard to jurisdictional claims in published maps and institutional affiliations.

\section{Submit your manuscript to a SpringerOpen ${ }^{\circ}$ journal and benefit from:}

- Convenient online submission

- Rigorous peer review

- Open access: articles freely available online

- High visibility within the field

- Retaining the copyright to your article

Submit your next manuscript at $\boldsymbol{\nabla}$ springeropen.com 